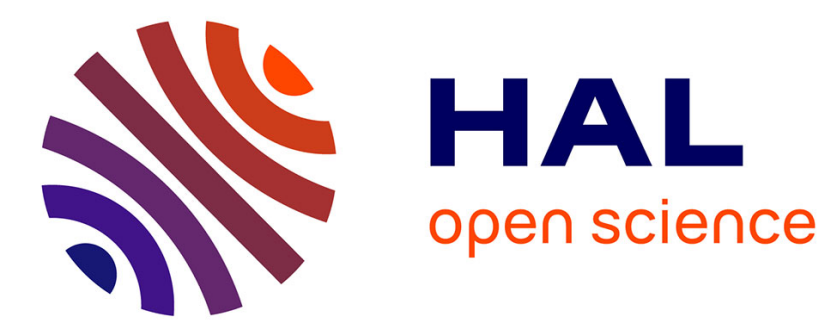

\title{
Optical angular momentum conversion in a nanoslit: comment
}

\author{
Etienne Brasselet
}

\section{To cite this version:}

Etienne Brasselet. Optical angular momentum conversion in a nanoslit: comment. Optics Letters, 2013, 38 (19), pp.3890. 10.1364/OL.38.003890 . hal-00880438

\section{HAL Id: hal-00880438 \\ https://hal.science/hal-00880438}

Submitted on 1 Mar 2018

HAL is a multi-disciplinary open access archive for the deposit and dissemination of scientific research documents, whether they are published or not. The documents may come from teaching and research institutions in France or abroad, or from public or private research centers.
L'archive ouverte pluridisciplinaire HAL, est destinée au dépôt et à la diffusion de documents scientifiques de niveau recherche, publiés ou non, émanant des établissements d'enseignement et de recherche français ou étrangers, des laboratoires publics ou privés.

\section{다)(1) $(5$}

Distributed under a Creative Commons Attribution - NonCommerciall 4.0 International 


\title{
Optical angular momentum conversion in a nanoslit: comment
}

\author{
Etienne Brasselet \\ Université de Bordeaux, CNRS, LOMA, UMR 5798, F 33400 Talence, France (e.brasselet@loma.u bordeaux1.fr)
}

\begin{abstract}
In a recent work [Opt. Lett. 37, 4946 (2012)], the spin to orbital optical angular momentum conversion from a sub wavelength slit having a circular shape has been reported. In particular, the conversion efficiency was claimed to be independent of the slit dichroism. Here, we correct such a statement and demonstrate that dichroism strongly influences the process of optical vortex generation.
\end{abstract}

OCIS codes: (050.4865) Optical vortices; (050.2555) Form birefringence; (310.6628) Subwavelength structures, nanostructures.

Recently, Chimento and coworkers investigated the effect of a circular subwavelength slit on the orbital angular momentum content of light [1] ]. A subwavelength slit indeed behaves as a birefringent retarder whose main axes are directed parallel and perpendicular to it [2]. This enables a circular slit to partially convert an incident circularly polarized light field into a contra-circularly polarized one carrying an on-axis optical phase singularity with a topological charge of two [1]. In particular, the efficiency $\eta$ of the polarization conversion process has been claimed to be independent of the slit dichroism [1].

The aim of this Comment is to correct the latter statement. Dichroism indeed strongly influences the process, as demonstrated hereafter in the more general framework of so-called $q$-plates [3], which consist of slabs of inhomogeneous and optically anisotropic slabs of thickness $L$ that have: (i) an azimuthal distribution of the orientation of their optical axis of the form $\psi(\phi)=q \phi+\phi_{0}$ with $q$ integer, $\phi$ the usual azimuthal angle in the $(x, y)$ plane of the slab, and $\phi_{0}$ a constant and (ii) a uniform birefringent phase retardation $\Delta=k_{0}\left(n_{\|}-n_{\perp}\right) L$ with $k_{0}$ the wavenumber in vacuum and $n_{\|, \perp}$ the refractive indices parallel and perpendicular to the optical axis.

Let us consider the case study of an incident circularly polarized plane wave that propagates through a dichroic $q$-plate of thickness $L$ with an input facet located at $z=0$ and with attenuation coefficients $\delta_{\|}=e^{-\alpha_{\|} L}$ and $\delta_{\perp}=e^{-\alpha_{\perp} L}$, where $\alpha_{\|}$and $\alpha_{\perp}$ are the amplitude attenuation constants for the polarizations parallel and perpendicular to the optical axis, respectively. The
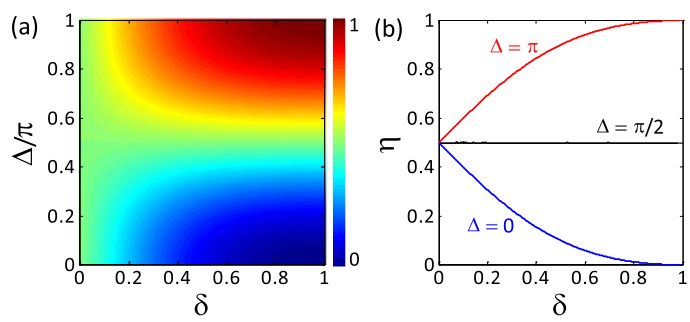

Fig. 1. (a) Map of polarization conversion efficiency $\eta$ as a function of birefringent phase retardation $\Delta$ and dichroism parameter $\delta$. (b) $\eta$ versus $\delta$ for $\Delta=0, \pi / 2$, and $\pi$, which corre spond to special cases of full wave, quarter wave, or half wave dichroic plates, respectively. incident electric field $(z<0)$ is expressed as $\mathbf{E}_{\text {in }}=$ $E_{0} e^{-i\left(\omega t-k_{0} z\right)} \mathbf{c}_{\sigma}$ where $\mathbf{c}_{\sigma}=(\mathbf{x}+i \sigma \mathbf{y}) / \sqrt{ } 2, \sigma= \pm 1$, refers to the orthonormal circular polarization basis. Neglecting diffraction effects, the output light field at $z=L$ is obtained in the laboratory frame by using the Jones formalism,

$$
\begin{aligned}
\mathbf{E}_{\text {out }}= & E_{0} e^{-i \omega t}\left(\begin{array}{cc}
\cos \psi & -\sin \psi \\
\sin \psi & \cos \psi
\end{array}\right)\left(\begin{array}{cc}
\delta_{\|} e^{i k_{0} n_{\|} L} & 0 \\
0 & \delta_{\perp} e^{i k_{0} n_{\perp} L}
\end{array}\right) \\
& \times\left(\begin{array}{cc}
\cos \psi & \sin \psi \\
-\sin \psi & \cos \psi
\end{array}\right) \mathbf{c}_{\sigma} .
\end{aligned}
$$

Expressing $\mathbf{E}_{\text {out }}$ in the circular polarization basis, one gets, up to a phase factor $e^{-i \omega t+i(\Delta / 2)+i k_{0} n_{\perp} L}$,

$$
\mathbf{E}_{\text {out }}=\frac{E_{0}}{2}\left[\left(\delta_{\|} e^{i \frac{\Delta}{2}}+\delta_{\perp} e^{-i \frac{\Delta}{2}}\right) \mathbf{c}_{\sigma}+\left(\delta_{\|} e^{i \frac{\Delta}{2}}-\delta_{\perp} e^{-i \frac{\Delta}{2}}\right) e^{i 2 \sigma \psi(\phi)} \mathbf{c}_{-\sigma}\right] .
$$

By introducing the dichroism parameter $\delta=\delta_{\perp} / \delta_{\|}$, if $\delta_{\perp}<\delta_{\|}$and $\delta=\delta_{\|} / \delta_{\perp}$ if $\delta_{\perp}>\delta_{\|}$, the efficiency $\eta$ is defined as the intensity ratio $\eta=\left(\left|\mathbf{E}_{\text {out }} \cdot \mathbf{c}_{-\sigma}^{*}\right|^{2}\right) /\left|\mathbf{E}_{\text {out }}\right|^{2}$, with the asterisk being a complex conjugation, expressed as

$$
\eta=\frac{(1-\delta)^{2}}{2\left(1+\delta^{2}\right)} \cos ^{2}(\Delta / 2)+\frac{(1+\delta)^{2}}{2\left(1+\delta^{2}\right)} \sin ^{2}(\Delta / 2)
$$

The spin-to-orbital optical angular momentum conversion thus strongly depends on the dichroism whatever is $q$, as illustrated in Fig. 1 . The "usual" formula $\eta=$ $\sin ^{2}(\Delta / 2)$ only applies without dichroism, i.e., $\delta=1$. For the case of $\delta=0$ in Fig. 1 , this is a special case of a linear polarizer with an azimuthally varying axis.

\section{References}

1. P. F. Chimento, P. F. A. Alkemade, G. W. 't Hooft, and E. R. Eliel, Opt. Lett. 37, 4946 (2012).

2. P. F. Chimento, N. V. Kuzmin, J. Bosman, P. F. A. Alkemade, G. W. 't Hooft, and E. R. Eliel, Opt. Express 19, 24219 (2011).

3. L. Marrucci, C. Manzo, and D. Paparo, Phys. Rev. Lett. 96, 163905 (2006). 